\title{
Moment-Ratio Diagrams for Univariate Distributions
}

\author{
ERIK VARGO \\ Department of Mathematics, The College of William \&3 Mary, Williamsburg, VA 23187
}

RAGHU PASUPATHY

The Grado Department of Industrial and Systems Engineering, Virginia Tech, Blacksburg, VA 24061

\section{LAWRENCE LEEMIS}

\author{
Department of Mathematics, The College of William \& Mary, Williamsburg, VA 23187
}

\begin{abstract}
We present two moment-ratio diagrams along with guidance for their interpretation. The first momentratio diagram is a graph of skewness versus kurtosis for common univariate probability distributions. The second moment-ratio diagram is a graph of coefficient of variation versus skewness for common univariate probability distributions. Both of these diagrams, to our knowledge, are the most comprehensive to date. The diagrams serve four purposes: (1) they quantify the proximity between various univariate distributions based on their second, third, and fourth moments; (2) they illustrate the versatility of a particular distribution based on the range of values that the various moments can assume; and (3) they can be used to create a short list of potential probability models based on a data set; (4) they clarify the limiting relationships between various well-known distribution families. The use of the moment-ratio diagrams for choosing a distribution that models given data is illustrated.
\end{abstract}

Key Words: Coefficient of Variation; Kurtosis; Skewness.

\section{Introduction}

$\mathbb{T}$ HE MOMENT-RATIO DIAGRAM for a distribution refers to the locus of a pair of standardized moments plotted on a single set of coordinate axes (Kotz and Johnson (2006)). By standardized moments we mean the coefficient of variation $(\mathrm{CV})$,

$$
\gamma_{2}=\frac{\sigma_{X}}{\mu_{X}}
$$

Mr. Vargo is a Ph.D. student at the University of Virginia. His email address is epvargo@wm.edu.

Dr. Pasupathy is an Assistant Professor in the Grado Department of Systems and Industrial Engineering. His email address is pasupath@vt.edu.

Dr. Leemis is a Professor in the Department of Mathematics. His email address is leemis@math.wm.edu. the skewness (or third standardized moment)

$$
\gamma_{3}=E\left[\left(\frac{X-\mu_{X}}{\sigma_{X}}\right)^{3}\right]
$$

and the kurtosis (or fourth standardized moment)

$$
\gamma_{4}=E\left[\left(\frac{X-\mu_{X}}{\sigma_{X}}\right)^{4}\right],
$$

where $\mu_{X}$ and $\sigma_{X}$ are the mean and the standard deviation of the implied (univariate) random variable $X$. The classical form of the moment-ratio diagram, plotted upside down, shows the third standardized moment $\gamma_{3}$ (or sometimes its square $\gamma_{3}^{2}$ ) plotted as abcissa and the fourth standardized moment $\gamma_{4}$ plotted as ordinate. The plot usually includes all possible pairs $\left(\gamma_{3}, \gamma_{4}\right)$ that a distribution can attain. Because $\gamma_{4}-\gamma_{3}^{2}-1 \geq 0$ (see Stuart and Ord (1994), Exercise 3.19 , p. 121), the moment-ratio diagram for a distribution occupies some subset of the shaded region shown in Figure 1. 


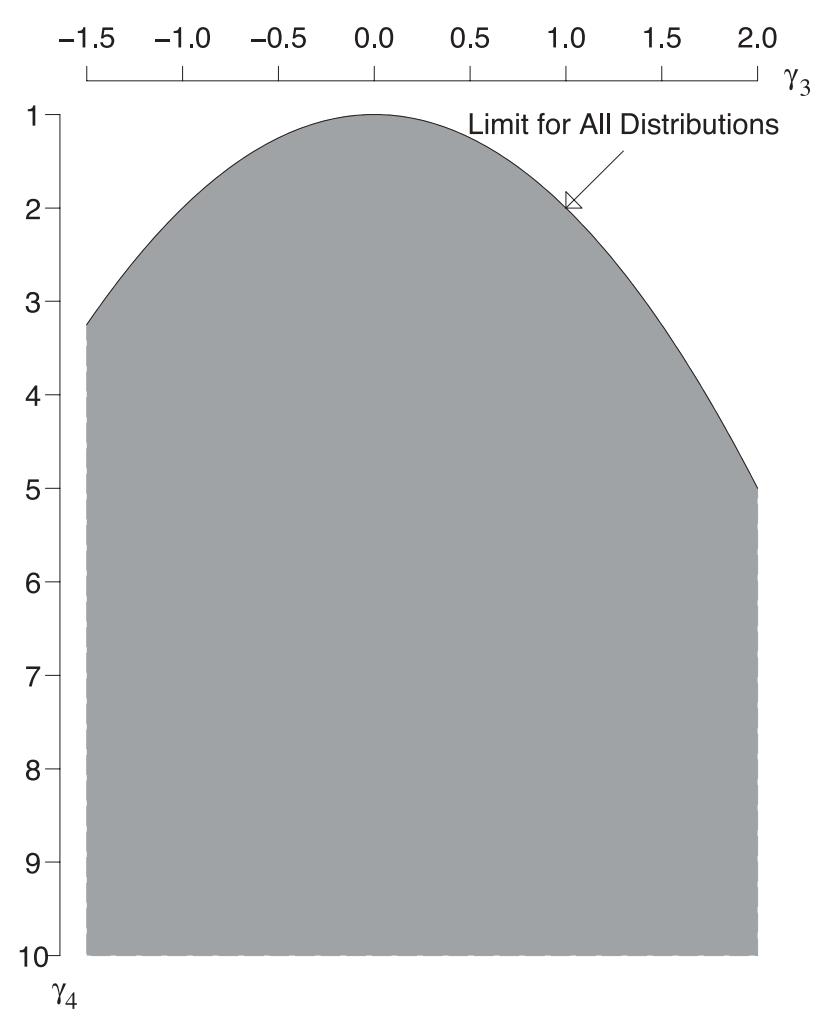

FIGURE 1. The Shaded Region Represents the Set of Attainable Pairs of Third and Fourth Standardized Moments $\left(\gamma_{3}, \gamma_{4}\right)$ for Any Distribution. The solid line is the limit $\gamma_{4}$ $=1+\gamma_{3}^{2}$ for all distributions.

Moment-ratio diagrams, apparently first introduced by Craig (1936) and later popularized by Johnson et al. (1994), especially through the plotting of multiple distributions on the same axes, have found enormous expediency among engineers and statisticians. The primary usefulness stems from the diagram's ability to provide a ready "snapshot" of the relative versatility of various distributions in terms of representing a range of shapes. Distributions occupying a greater proportion of the moment-ratio region are thought to be more versatile owing to the fact that a larger subset of the allowable moment pairs can be modeled by the distribution. Accordingly, when faced with the problem of having to choose a distribution to model given data, modelers often estimate the third and fourth standardized moments (along with their standard error estimates) and plot them on a moment-ratio diagram to get a sense of which distributions may be capable of representing the shapes implicit in the provided data. In this sense, a modeler can compare several "candidate" distributions simultaneously in terms of their moments. Another use for these diagrams has been in getting a sense of the limiting relationships between distributions, and also between various distributions within a system. An excellent example of the latter is the Pearson system of frequency curves where the region occupied by the various distributions comprising the system neatly divides the $\left(\gamma_{3}^{2}, \gamma_{4}\right)$ plane (Johnson et al. (1994)).

Since Craig (1936) published the original momentratio diagram, various authors have expanded and published updated versions. The most popular of these happen to be the various diagrams appearing in Johnson et al. ((1994), pp. 23, 390). Rodriguez (1977), in clarifying the region occupied by the Burr Type XII distribution in relation to others, provides a fairly comprehensive version of the moment-ratio diagram showing several important regions. Tadikamalla (1980) provides a similar but limited version in clarifying the region occupied by the Burr Type III region.

More recently, Cox and Oakes (1984) have popularized a moment-ratio diagram of a different kindone that plots the $\mathrm{CV}\left(\gamma_{2}\right)$ as the abcissa and the third standardized moment $\left(\gamma_{3}\right)$ as the ordinate. Admittedly, this variation is location and scale dependent, unlike the classical moment-ratio diagrams involving the third and fourth standardized moments. Nevertheless, the diagram has become unquestionably useful for modelers. A slightly expanded version of this variation appears in Meeker and Escobar (1998).

\section{Contribution}

Our contributions in this paper are threefold, stated here in order of importance. First, we provide a moment-ratio diagram of the $\mathrm{CV}$ versus skewness $\left(\gamma_{2}, \gamma_{3}\right)$ involving 36 distributions, four of which occupy two-dimensional regions within the plot. To our knowledge, this is the most comprehensive diagram available to date. Furthermore, it is the first time the entire region occupied by important two-parameter families within the CV versus skewness plot (e.g., generalized gamma, beta, Burr Type XII) has been calculated and depicted. The CV versus skewness plot first appeared in Cox and Oakes (1984) and later in Meeker and Escobar (1998). The diagrams appearing in both these original sources either depict only families with a single shape parameter (e.g., gamma) or vary only one of the shape parameters while fixing all others. Second, we provide a classical momentratio diagram $\left(\gamma_{3}, \gamma_{4}\right)$ that includes 37 distributions, 
four of which occupy two-dimensional regions within the plot. While such diagrams are widely available, the diagram we provide is the most comprehensive among the sources we know and seems particularly useful due to its depiction of all distributions in the same plot. In constructing the two moment-ratio diagrams, we have had to derive the limiting behavior of a number of distributions, some of which seem to be new. Expressions for $\gamma_{2}, \gamma_{3}$, and $\gamma_{4}$ for some of these distributions are listed in the Appendix. We also host the moment-ratio diagrams in a publicly accessible website where particular regions of the diagram can be magnified for clearer viewing. Third, using an actual data set, we demonstrate what a modeler might do when having to choose candidate distributions that "model" given data.

\section{Organization of the Paper}

The rest of the paper is organized as follows. We present the two moment-ratio diagrams along with cues for interpretation in the next section. Following that, we demonstrate the use of the momentratio diagrams for choosing a distribution that models given data. Finally, we present conclusions and suggestions for further research. This is followed by the Appendix, where we provide analytical expressions for the moment-ratio locus corresponding to some of the distributions depicted in the diagrams.

\section{Reading the Moment-Ratio Diagrams}

Two moment-ratio diagrams are presented in this paper. The first, shown in Figure 2, is a plot containing the $\left(\gamma_{3}, \gamma_{4}\right)$ regions for 37 distributions. Figure 3 is a plot containing the $\left(\gamma_{2}, \gamma_{3}\right)$ regions for 36 distributions. For convenience, in both diagrams, we have chosen to include discrete and continuous distributions on the same plot. In what follows, we provide a common list of cues that will be useful in reading the diagrams correctly.

(i) Distributions of which moment-ratio regions correspond to single points (e.g., normal) are represented by black solid dots, curves (e.g., gamma) are represented by solid black lines, and areas (e.g., Burr Type XII) are represented by colored regions.

(ii) The names of continuous distributions occupying a region are set in sans serif type; the names of continuous distributions occupying a point or curve are set in roman type; the names of discrete distributions occupying a point or curve are set in italic type.

(iii) The end points of curves, when not attained by the distribution in question, are represented by an unfilled circle (e.g., logistic exponential).

(iv) When the boundary of a moment-ratio area is obscured by another area, we include a dotted line (Figure 2) or an arrow (Figure 3) to clarify the location of the obscured boundary.

(v) When a distribution represented by points in one of the moment-ratio diagrams converges as one of its parameters approaches a limiting value (e.g., a $t$ random variable as its degrees of freedom approaches infinity), we often decrease the font size of the labels to minimize interference.

(vi) The parameterizations used for the distributions are from Leemis and McQueston (2008) unless indicated otherwise in the paper.

\section{The Skewness-Kurtosis Diagram}

Whether the locus corresponding to a distribution in Figure 2 is a point, curve, or region usually depends on the number of shape parameters. For example, the normal distribution has no shape parameters and its locus in Figure 2 corresponds to the point $(0,3)$. By contrast, because the gamma distribution has one shape parameter, its locus corresponds to the curve $\gamma_{3}=1.5 \gamma_{2}^{2}+3$. An example of a distribution that has two shape parameters is the Burr Type XII distribution. It accordingly occupies an entire region in Figure 2. In all, Figure 2 has 37 distributions with 4 continuous distributions represented by regions, 19 distributions (15 continuous and 4 discrete) represented by curves, and 14 distributions (13 continuous and 1 discrete) represented by one or more points. A list of other useful facts relating to Figure 2 follows.

- The "T" plotted at $\left(\gamma_{3}, \gamma_{4}\right)=(0,9)$ corresponds to the $t$-distribution with five degrees of freedom, which is the smallest number of degrees of freedom where the kurtosis exists.

- The chi-square (S) and Erlang (X) distributions coincide when the chi-square distribution has an even number of degrees of freedom. This accounts for the alternating pattern of "S" and "SX" labels that occur along the curve associated with the gamma distribution.

- Numerous distributions start at (or include) the locus of the normal distribution and end at (or include) the locus of the exponential distribution. Two examples of such are the gamma distribution and the inverted beta distribution.

- Space limitations prevented us from plotting the values associated with the discrete uniform- 

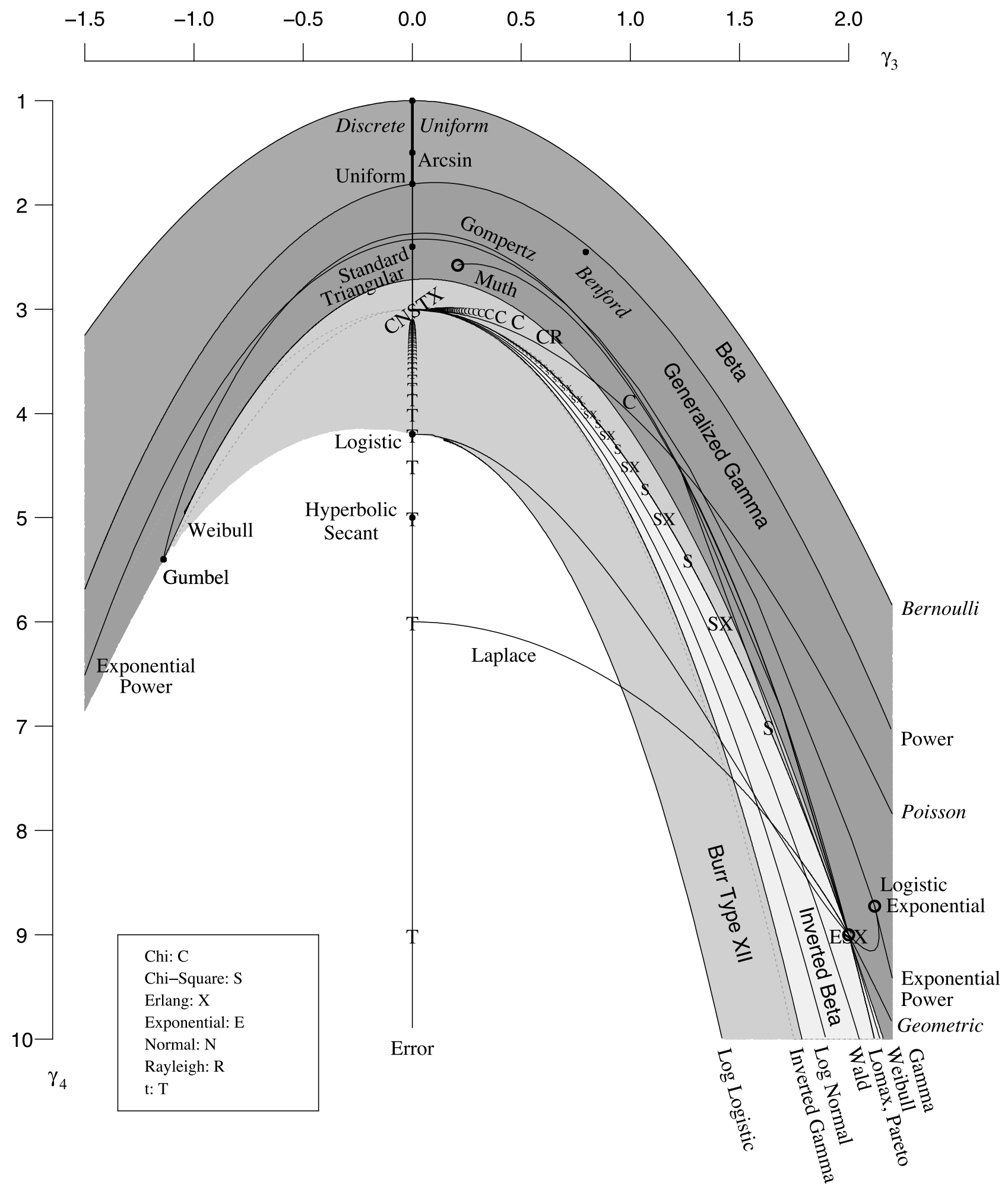

FIGURE 2. Skewness $\left(\gamma_{3}\right)$ Versus Kurtosis $\left(\gamma_{4}\right)$. (See the supplementary material at http://www.asq.org/pub/jqt/ for a full-color version of this figure.) 


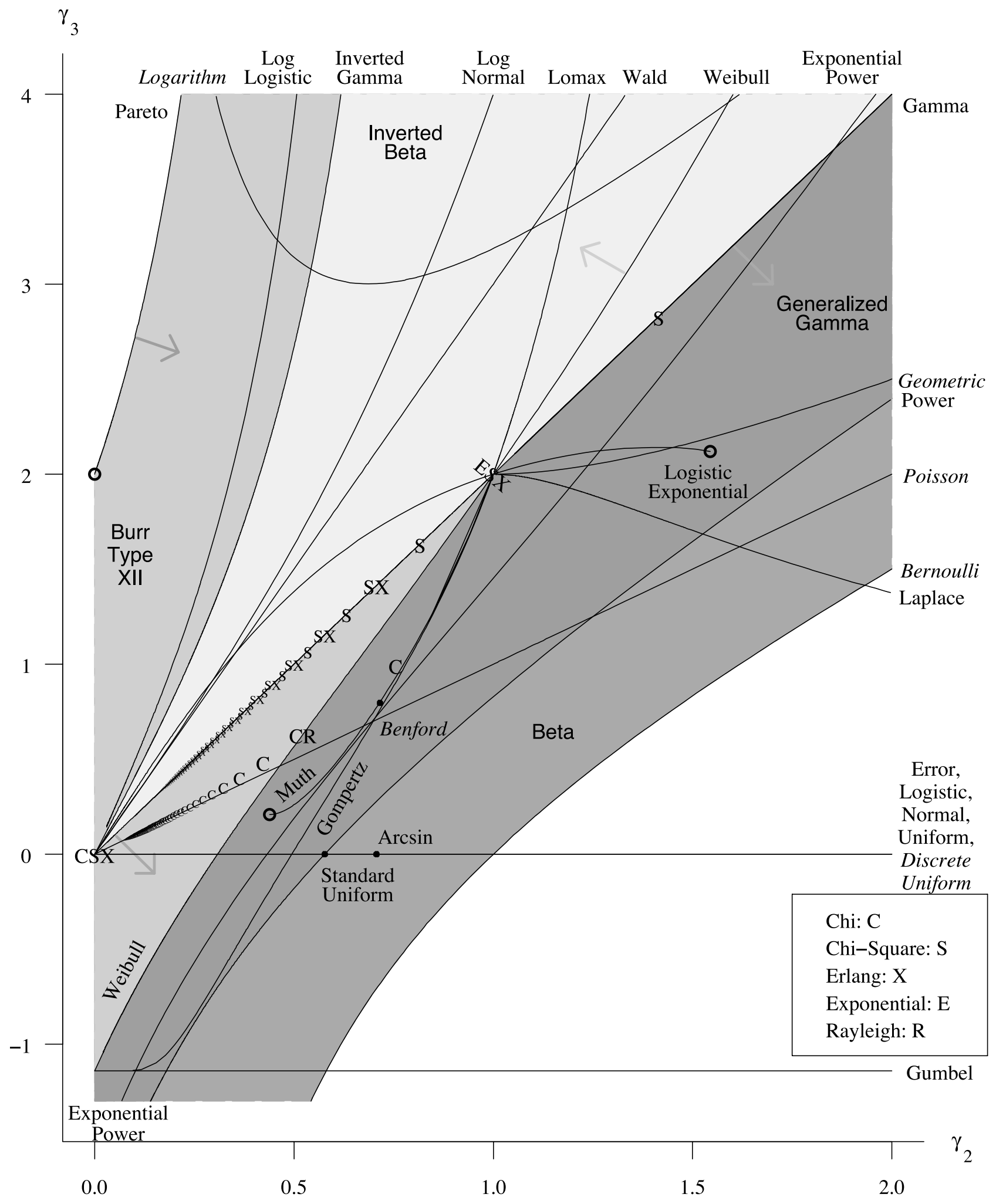

FIGURE 3. CV $\left(\gamma_{2}\right)$ Versus Skewness $\left(\gamma_{3}\right)$. (See the supplementary material at http://www.asq.org/pub/jqt/ for a full-color version of this figure.) 
distribution between its limits as a two-mass value with $\left(\gamma_{3}, \gamma_{4}\right)=(0,1)$ and its limiting distribution (as the number of mass values increases $)$ with $\left(\gamma_{3}, \gamma_{4}\right)=(0,1.8)$. It is plotted as a thick line.

- The regime occupied by the inverted beta distribution has the curves corresponding to inverted gamma and the gamma distributions as limits.

- The regime occupied by the generalized gamma distribution has the curves corresponding to the power distribution and the log gamma distribution as partial limits.

- The regime occupied by the Burr Type XII distribution has the curve corresponding to the Weibull distribution as a partial limit.

- Barring extreme negative skewness values, virtually all of the regime occupied by the generalized gamma distribution is subsumed by the beta distribution.

- The beta and the Burr Type XII distributions seem complementary in the sense that the beta distribution occupies the "outer" regions of the diagram while the Burr Type XII distribution occupies the "inner" regions of the diagram. Furthermore, the collective regime of the beta and Burr Type XII distributions, with a few exceptions (e.g., Laplace), encompasses all other distributions included in the plot.

\section{The CV-Skewness Diagram}

Unlike in the skewness-kurtosis diagram (Figure 2 ), the locus of a distribution in the CV-skewness diagram (Figure 3) depends on the distribution's location and scale parameters. For this reason, in Figure 3, there are fewer distributions (compared with Figure 2) for which the locus is a singleton. Figure 3 represents a total of 36 distributions with 4 continuous distributions represented by regions, 24 distributions (19 continuous and 5 discrete) represented by curves, and 8 distributions ( 7 continuous and 1 discrete) represented by one or more points. A list of other useful facts relating to Figure 3 follows.

- Distributions that are symmetric about the mean have $\gamma_{3}=0$. Because CV can be adjusted to take any value (by controlling the location and scale), symmetric distributions, e.g., error, normal, uniform, logistic, have the locus $\gamma_{3}=0$ in Figure 3.

- The regime occupied by the beta family has the gamma curve $\gamma_{3}=2 \gamma_{2}, \gamma_{2} \in(0,1)$ and the
Bernoulli curve $\gamma_{3}=\gamma_{2}-1 / \gamma_{2}$ as limits.

- The regime occupied by the inverted beta distribution has the gamma curve $\gamma_{3}=2 \gamma_{2}$, $\gamma_{2} \in(0,1)$ and the inverted gamma curve $\gamma_{3}=4 \gamma_{2} /\left(1-\gamma_{2}^{2}\right), \gamma_{2} \in(0,1)$ as limits.

- The regime occupied by the generalized gamma distribution has the curves corresponding to the power distribution and the Pareto distribution as partial limits.

- The regime occupied by the Burr Type XII distribution has the curves corresponding to the Weibull and Pareto distributions as limits.

\section{Application}

The moment-ratio diagrams can be used to identify likely candidate distributions for a data set, particularly through a novel use of bootstrapping techniques, e.g., Cheng (2006) and Ross (2006). Toward illustrating this, we first formally set up the problem. Let $X_{1}, X_{2}, \ldots, X_{n}$ be i.i.d. observations of a random variable having an unknown cumulative distribution function (CDF) $F(x)$. Suppose $\theta$ is some parameter concerning the population distribution (e.g., the coefficient of variation $\gamma_{2}$ ), and let $\hat{\theta}$ be its estimator (e.g., the sample coefficient of variation $\hat{\gamma}_{2}$ constructed from $\left.X_{1}, X_{2}, \ldots, X_{n}\right)$. Also let $F_{n}(x)$ denote the usual empirical CDF constructed from the data $X_{1}, X_{2}, \ldots, X_{n}$, i.e.,

$$
F_{n}(x)=\frac{1}{n} \sum_{i=1}^{n} I\left\{X_{i} \leq x\right\} .
$$

A lot is known about about how well $F_{n}(x)$ approximates $F(x)$. For example, the Glivenko-Cantelli theorem (Billingsley (1995)) states that $F_{n} \rightarrow F$ uniformly in $x$ as $n \rightarrow \infty$. Furthermore, the deviation of $F_{n}(x)$ from $F(x)$ can be characterized fully through Sanov's theorem (Dembo and Zeitouni (1998)) under certain conditions.

We are now ready to demonstrate how the above can be used toward identifying candidate distributions to which a given set of data $X_{1}, X_{2}, \ldots, X_{n}$ might belong. As usual, the sample mean and sample standard deviation are calculated as

$$
\bar{X}=\frac{1}{n} \sum_{i=1}^{n} X_{i} \quad \text { and } \quad S=\sqrt{\frac{1}{n-1} \sum_{i=1}^{n}\left(X_{i}-\bar{X}\right)^{2}} .
$$

In order to obtain a nonzero standard deviation, we assume that at least two of the data values are distinct. The point estimates for the CV, skewness, and 
kurtosis are

$$
\begin{aligned}
& \hat{\gamma}_{2}=\frac{S}{\bar{X}}, \\
& \hat{\gamma}_{3}=\frac{1}{n} \sum_{i=1}^{n}\left(\frac{X_{i}-\bar{X}}{S}\right)^{3}, \\
& \hat{\gamma}_{4}=\frac{1}{n} \sum_{i=1}^{n}\left(\frac{X_{i}-\bar{X}}{S}\right)^{4},
\end{aligned}
$$

for $\bar{X} \neq 0$. The points $\left(\hat{\gamma}_{3}, \hat{\gamma}_{4}\right)$ and $\left(\hat{\gamma}_{2}, \hat{\gamma}_{3}\right)$ can be plotted in Figures 2 and 3 to give a modeler guidance concerning which distributions are potential parametric models for statistical inference. Probability distributions in the vicinity of the point estimates are strong candidates for probability models. Unfortunately, these point estimates do not give the modeler a sense of their precision, so we develop an approximate interval estimate in the paragraph below.

Bootstrapping can be used to obtain a measure of the accuracy of the point estimates $\left(\hat{\gamma}_{3}, \hat{\gamma}_{4}\right)$ and $\left(\hat{\gamma}_{2}, \hat{\gamma}_{3}\right)$. Let $B$ denote the number of bootstrap samples (a bootstrap sample consists of $n$ observations drawn with replacement from the original data set). For each bootstrap sample, the two parameters of interest (e.g., skewness and kurtosis) are estimated using the procedure described in the previous paragraph and stored. After the $B$ bootstrap samples have been calculated, the bivariate normal distribution is fitted to the $B$ data pairs using standard techniques. Two of the five parameters of the bivariate normal distribution, namely, the two sample bootstrap means, are replaced by the point estimators to assure that the bivariate normal distribution is centered about the point estimators that were calculated and plotted in the previous paragraph. Finally, a concentration ellipse is plotted around the point estimate. The tilt associated with the concentration ellipse gives the modeler a sense of the correlation between the two parameters of interest.

\section{Example}

Consider the $n=23$ deep-groove ball-bearing failure times (measured in $10^{6}$ revolutions)

$\begin{array}{rrrrrr}17.88 & 28.92 & 33.00 & 41.52 & 42.12 & 45.60 \\ 48.48 & 51.84 & 51.96 & 54.12 & 55.56 & 67.80 \\ 68.64 & 68.64 & 68.88 & 84.12 & 93.12 & 98.64 \\ 105.12 & 105.84 & 127.92 & 128.04 & 173.40 & \end{array}$

from Lieblein and Zelen (1956), which is discussed in Caroni (2002). For brevity, we consider the plotting of the point and associated concentration ellipse for only the $\mathrm{CV}$ versus skewness moment ratio diagram
(Figure 3). The first step is to calculate and plot the point $\left(\hat{\gamma}_{2}, \hat{\gamma}_{3}\right) \cong(0.519,0.881)$. We then take $B=200$ bootstrap samples of $n=23$ failure times with replacement from the data set. (The value of $B$ was chosen arbitrarily.) The bivariate normal distribution is fitted to the $B$ data pairs and a concentration ellipse is then overlaid on the plot of the $\mathrm{CV}$ versus skewness as a visual aid to identify likely candidate distributions for modeling the ball-bearing lifetimes. The results of this process are displayed in Figure 4, which provides a close-up view of the concentration ellipse. In terms of candidate distributions, the following conclusions can be drawn.

- Because ball-bearing lifetimes are inherently continuous, all of the discrete distributions should be eliminated from consideration.

- The position of the concentration ellipse implies that several distributions associated with regions in the $\left(\gamma_{2}, \gamma_{3}\right)$ graph are candidate distributions: the gamma distribution (and its special cases), and the Weibull distribution (and the Rayleigh distribution as a special case) are likely to be models that fit the data well.

- The gamma and Weibull distributions both have shape parameters that are greater than 1 within the concentration ellipse, confirming the intuition that an appropriate model is in the increasing failure rate (IFR) class (Cox and Oakes (1984)) of survival distributions (i.e., the ball bearings are wearing out). Consistent with this conclusion, notice that the point for the exponential distribution is far away from the concentration ellipse.

- Distributions that are close to the concentration ellipse should also be included as candidates. For this data set, the log-normal distribution is just outside of the concentration ellipse, but provides a good fit to the data (see Crowder et al. (1991), pp. 37-38 and 42-43 for details). Any distribution in or near the concentration ellipse should be considered a candidate distribution. This is confirmed by the four graphs in Figure 5, which show the fitted Weibull, gamma, log-normal, and exponential distributions, along with the empirical CDF associated with the ball-bearing failure data. The three distributions that are inside of or close to the concentration ellipse provide reasonable fits to the data; the exponential distribution, which is far away from the concentration ellipse, provides a poor fit to the data. 


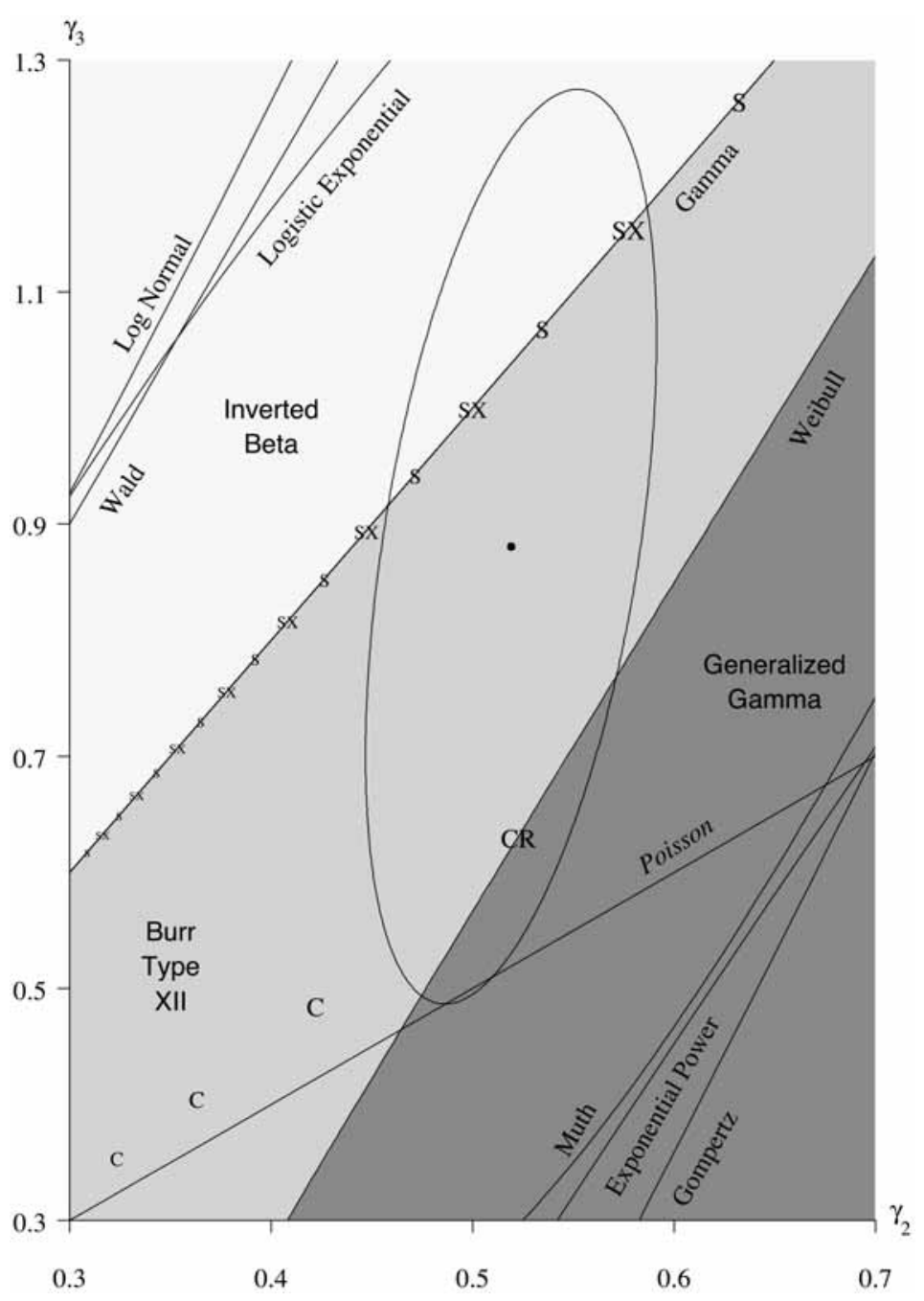

FIGURE 4. Using the CV-Skewness Diagram to Choose a Candidate Distribution (for Modeling Given Data) Through Estimation and Bootstrapping.

The size of the concentration ellipse also gives guidelines with respect to sample size. If the concentration ellipse is so large that dozens of probability distributions are viable candidates, then a larger sample size is required. As expected, there is generally more variability on the higher-order moments.

Also, the eccentricity and tilt of the concentration ellipse provide insight on the magnitudes of the variances of the point estimates and their correlation. For the ball-bearing failure times, the standard error of the skewness is almost an order of magnitude larger than the standard error of the coefficient of variation. The slight tilt of the concentration ellipse indicates that there is a small positive correlation between the coefficient of variation and the skewness.
If point estimates and concentration ellipses are plotted on both of the moment-ratio diagrams in Figures 2 and 3 , the candidate distributions might not be consistent. The authors believe that the coefficient of variation versus the skewness plot is more reliable because it is based on lower order moments. The moment-ratio diagrams can be used in tandem when using any one diagram still leaves a large number of candidate distributions.

\section{Conclusions and Further Research}

The two moment-ratio diagrams presented in Figures 2 and 3 are useful for insight concerning univariate probability distributions and for model discrimination for a particular data set. Plotting a concen- 

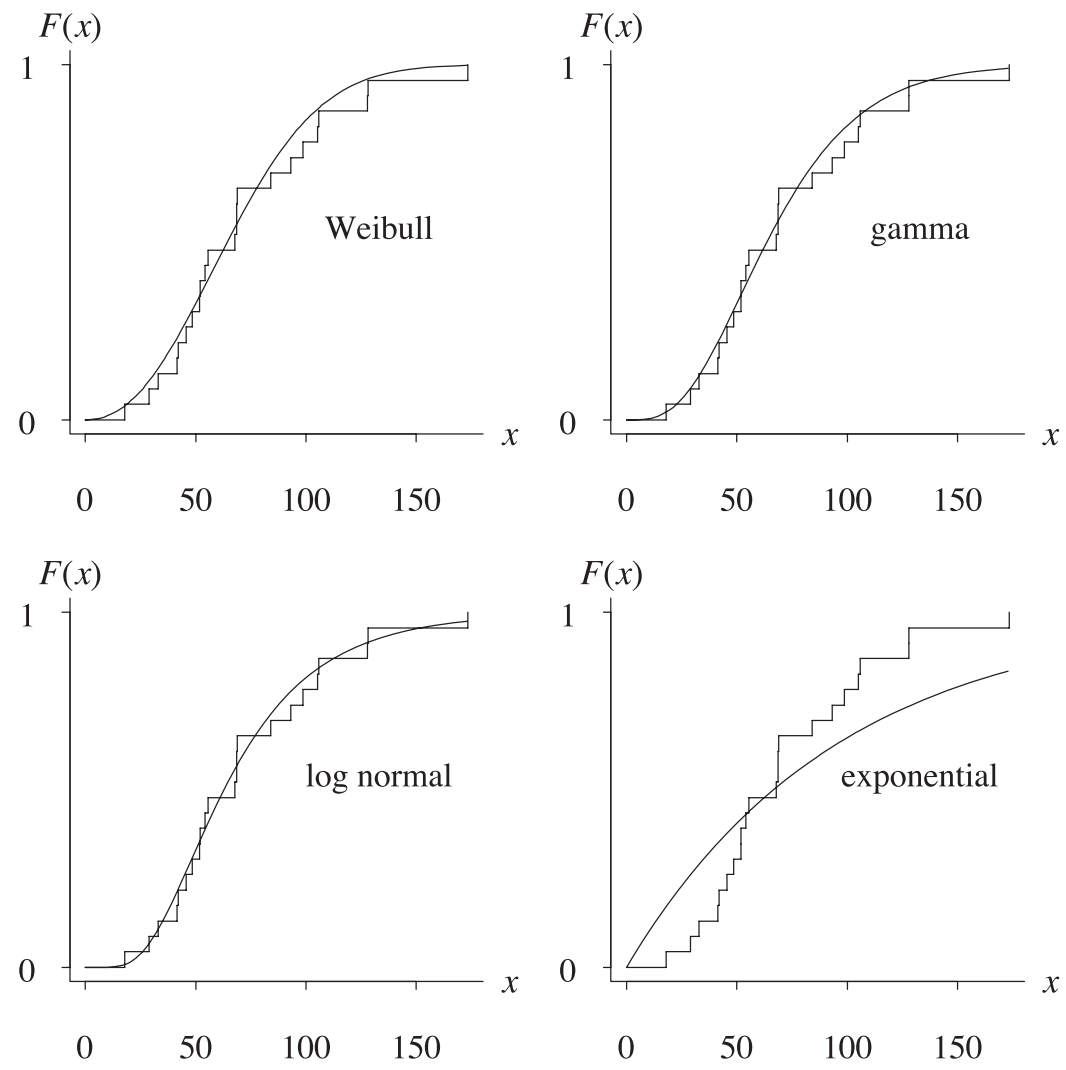

FIGURE 5. Empirical and Fitted CDFs for the Weibull, Gamma, Log-Normal, and Exponential Distributions.

tration ellipse associated with bootstrap samples on either chart provides guidance concerning potential probability distributions that provide an adequate fit to a data set. These diagrams are one of the few ways that data analysts can simultaneously evaluate multiple univariate distributions.

Data sets associated with actuarial science, biostatistics, and reliability engineering often contain censored observations, with right-censored observations being the most common. Plotting the various moments is problematic for censored observations. Block and Leemis (2008) provide techniques for overcoming censoring that are based on kernel density function estimation and competing risks theory. These techniques can be adapted to produce point estimators and concentration ellipses.

Further research work associated with these diagrams would include a Monte Carlo study that evaluates the effectiveness of the concentration ellipse in identifying candidate distributions. This study would indicate which of the two moment-ratio diagrams is better for model discrimination.

\section{Appendix}

In this section, we provide exact expressions for the $\mathrm{CV}$, skewness, and kurtosis for the four distributions that occupy (two-dimensional) regions in Figures 2 and 3 .

\section{Beta}

The beta family (Johnson et al. (1995), p. 210) has two shape parameters, $p, q>0$, with

$$
\begin{aligned}
& \gamma_{2}=\frac{\sqrt{q}}{\sqrt{p^{2}+p q+p}}, \quad p, q>0 ; \\
& \gamma_{3}=\frac{2(q-p) \sqrt{1 / p+1 / q+1 / p q}}{p+q+2}, \quad p, q>0 ; \\
& \gamma_{4}=3(p+q+1) \frac{2(p+q)^{2}+p q(p+q-6)}{p q(p+q+2)(p+q+3)}, \\
& p, q>0 .
\end{aligned}
$$

The regime in the $\left(\gamma_{2}, \gamma_{3}\right)$ plane is bounded above by the line $\gamma_{3}=2 \gamma_{2}$ corresponding to the gamma family, and below by the curve $\gamma_{3}=\gamma_{2}-1 / \gamma_{2}$. The regime in the $\left(\gamma_{3}, \gamma_{4}\right)$ plane is bounded below by the limiting curve $\gamma_{4}=1+\gamma_{3}^{2}$ for all distributions and 
above by the curve $\gamma_{4}=3+(3 / 2) \gamma_{3}^{2}$ corresponding to the gamma family.

\section{Inverted Beta}

The beta-prime or the Pearson Type VI family (Johnson et al. (1995), p. 248), also known as the inverted beta family, has two shape parameters, $\alpha, \beta>0$, with

$$
\begin{aligned}
\gamma_{2} & =\sqrt{\frac{\alpha+\beta-1}{\alpha(\beta-2)}}, \quad \beta>2 ; \\
\gamma_{3} & =\sqrt{\frac{4(\beta-2)}{(\alpha+\beta-1) \alpha} \cdot \frac{2 \alpha+\beta-1}{\beta-3}, \quad \beta>3 ;} \\
\gamma_{4} & =\frac{3\left(\alpha-2+\frac{1}{2}(\beta-3) \gamma_{2}^{2}\right)}{\beta-4}, \quad \beta>4 .
\end{aligned}
$$

The regime in the $\left(\gamma_{2}, \gamma_{3}\right)$ plane is bounded above by the curve $\gamma_{3}=4 \gamma_{2} /\left(1-\gamma_{2}^{2}\right), \gamma_{2} \in(0,1)$ and below by the curve $\gamma_{3}=2 \gamma_{2}$ corresponding to the gamma family. The regime in the $\left(\gamma_{3}, \gamma_{4}\right)$ plane is bounded above by the curve

$\gamma_{3}=\frac{4 \sqrt{\alpha-2}}{\alpha-3}, \quad \gamma_{4}=3+\frac{30 \alpha-66}{(\alpha-3)(\alpha-4)}, \quad \alpha>4$

corresponding to the inverse gamma family and below by the curve $\gamma_{4}=3+\frac{3}{2} \gamma_{3}^{2}$ corresponding to the gamma family.

\section{Generalized Gamma}

The generalized gamma family (Johnson et al. (1994), p. 388) has two shape parameters, $\alpha, \lambda>0$, with the $r$ th raw moment $\mu_{r}^{\prime}=\Gamma(\alpha+r \lambda) / \Gamma(\alpha)$. The regime in the $\left(\gamma_{2}, \gamma_{3}\right)$ plane is bounded below by the curve

$\gamma_{2}=\frac{1}{\sqrt{p(p+2)}}, \quad \gamma_{3}=\frac{1-p}{p+3} \cdot \frac{2}{\sqrt{1+2 / p}}, \quad p>0$

corresponding to the power family and above by the curve

$$
\gamma_{2}=\frac{1}{\sqrt{p(p-2)}}, \quad \gamma_{3}=\frac{1+p}{p-3} \cdot \frac{2}{\sqrt{1-2 / p}}, \quad p>3
$$

corresponding to the Pareto family. The regime in the $\left(\gamma_{3}, \gamma_{4}\right)$ plane is bounded above by the curve

$$
\begin{aligned}
\gamma_{3} & =\frac{1+p}{p-3} \frac{2}{\sqrt{1-2 / p}}, \\
\gamma_{4} & =\frac{3(1+2 / p)\left(3 p^{2}-p+2\right)}{(p+3)(p+4)}, \quad p>0
\end{aligned}
$$

corresponding to the power family, bounded below to the right by the curve corresponding to the generalized gamma family with $\lambda=-0.54$, and bounded below to the left by the curve corresponding to the log gamma family. (Recall that the log gamma family with shape parameter $\alpha>0$ has the $r$ th cumulant $\kappa_{r}=\Psi^{(r)}(\alpha)$, where $\Psi^{(r)}(z)$ is the $(r+1)$ th derivative of $\ln \Gamma(z)$.)

\section{Burr Type XII}

The Burr Type XII family (Rodriguez (1977)) has two shape parameters, $c, k>0$, with the $r$ th raw moment $\mu_{r}^{\prime}=\Gamma(r / c+1) \Gamma(k-r / c) / \Gamma(k), c>0, k>$ $0, r<c k$. The regime in the $\left(\gamma_{2}, \gamma_{3}\right)$ plane is bounded below by the curve corresponding to the Weibull family $\left(r\right.$ th raw moment $\mu_{r}^{\prime}=\Gamma(r / c+1)$, where $c>0$ is the Weibull shape parameter), and above by the curve

$\gamma_{2}=\frac{1}{\sqrt{p(p-2)}}, \quad \gamma_{3}=\frac{1+p}{p-3} \cdot \frac{2}{\sqrt{1-2 / p}}, \quad p>3$ corresponding to the Pareto family. The regime in the $\left(\gamma_{3}, \gamma_{4}\right)$ plane is bounded below by the curve corresponding to the Weibull family, bounded above to the right by the curve corresponding to the Burr Type XII family with $k=1$, and bounded above to the left by the curve corresponding to the Burr Type XII family with $c=\infty$.

\section{References}

Billingsley, P. (1995). Probability and Measure. New York, NY: Wiley-Interscience.

Block, A. D. and Leemis, L. M. (2008). "Model Discrimination for Heavily Censored Survival Data". IEEE Transactions on Reliability 57, pp. 248-259.

Caroni, C. (2002). "The Correct 'Ball Bearings' Data". Lifetime Data Analysis 8, pp. 395-399.

Cheng, R. C. H. (2006). "Resampling Methods". In Simulation, S. Henderson and B. L. Nelson, Eds. Handbooks in Operations Research and Management Science, pp. 415-453. Amsterdam, The Netherlands: Elsevier.

Cox, D. R. and OAkes, D. (1984). Analysis of Survival Data. United Kingdom, Chapman \& Hall/CRC.

Craig, C. C. (1936). "A New Exposition and Chart for the Pearson System of Frequency Curves". Annals of Mathematical Statistics 7, pp. 16-28.

Crowder, M. J.; Kimber, A. C.; Smith, R. L.; and SweetING, T. J. (1991). Statistical Analysis of Reliability Data. New York, NY: Chapman and Hall.

Dembo, A. and Zeitouni, O. (1998). Large Deviations Techniques and Applications. New York, NY: Springer-Verlag.

Johnson, N. L.; Kotz, S.; and Balakrishnan, N. (1994). Continuous Univariate Distributions, Volume 1. New York, NY: John Wiley \& Sons, Inc.

Johnson, N. L.; Kotz, S.; and BALAKRIShNAN, N. (1995). Continuous Univariate Distributions, Volume 2. New York, NY: John Wiley \& Sons, Inc.

Kotz, S. and Johnson, N. L. (2006). Encyclopedia of Statistical Sciences, Volume 5. New York, NY: Wiley-Interscience. 
Leemis, L. M. and McQueston, J. T. (2008). "Univariate Distribution Relationships". The American Statistician 62, pp. $45-53$.

Lieblein, J. and Zelen, M. (1956). "Statistical Investigation of the Fatigue Life of Deep-Groove Ball Bearings". Journal of Research of the National Bureau of Standards 57, pp. 273316.

Meeker, W. Q. and Escobar, L. A. (1998). Statistical Methods for Reliability Data. New York, NY: Wiley-Interscience.
Rodriguez, R. N. (1977). "A Guide to the Burr Type XII Distributions". Biometrika 64, pp. 129-134.

Ross, S. M. (2006). Simulation. Burlington, MA: Elsevier Academic Press.

Stuart, A. and Ord, K. (1994). Kendall's Advanced Theory of Statistics, Volume 1. New York, NY: Hodder Arnold.

Tadikamalla, P. R. (1980). "A Look at the Burr and Related Distributions". International Statistical Review 48, pp. 337344 . 